\title{
Stimulation-produced analgesia and suppression of behavior in the rat
}

\author{
AGNES J. THOMAS \\ Case Western Reserve University, Cleveland, Ohio
}

\begin{abstract}
Electrical stimulation of brain sites close to the cerebral aqueduct and third ventricle, which produces analgesia, can also produce a strong suppression of behavior. Unlike analgesia, the suppression was insensitive to naloxone blockade, and could be obtained independently of analgesia by stimulation at sites in the dorsal periaqueductal gray. This suggests the presence of an endogenous tranquilizing system in the midbrain of the rat, which may play a role in recuperative behaviors following a painful experience.
\end{abstract}

Recently, a reduction in pain sensitivity produced by brain stimulation, often referred to as stimulationproduced analgesia (SPA), has been demonstrated in vertebrate animals (e.g., Geisler \& Liebeskind, 1976; Liebeskind, Guilband, Besson, \& Oliveras, 1973), including humans (e.g., Hosobuchi, Adams, \& Linchitz, 1977; Richardson \& Akil, 1977a, 1977b). SPA and morphine analgesia share comparable physiological effects, including similar analgesic potency (Mayer, 1980), the development of tolerance and cross-tolerance (Mayer \& Hayes, 1975 ), and reversibility with the narcotic antagonist naloxone (Narcan) in rats (Akil, Mayer, \& Liebeskind, 1976), cats (Liebeskind et al., 1973), and humans (Adams, 1976). It is believed that SPA is obtained through the release of endogenous opiates (Hughes, Smith, \& Kosterlitz, 1975) that bind to specialized receptors (Pert \& Snyder, 1973a, 1973b) found along known pain fibers (Bowsher, 1957; Brodal, 1981) and pain pathways (Anderson, Basbaum, \& Fields, 1977; Basbaum, Clanton, \& Fields, 1976) in vertebrates. Additional studies have identified three endogenous opioid gene families, Proopiomelanocortin, Pro-enkephalin, and Prodynorphin, which are "heavily invested in systems that regulate the body's responses to stress" (Akil, Watson, Lewis, Khachaturian, \& Walker, 1984). Further, it has been found that central nuclei that regulate autonomic function (e.g., the nucleus tractus solitarius) are "enriched with endogenous opioids and their receptors" (Akil et al., 1984). Although low doses of endorphins can have an activational effect on behavior in mice (Katz, Carroll, \& Baldright, 1978), higher doses of these peptides can produce analgesia and sedation (Jaquet \& Marks, 1976). Both opioid and nonopioid forms of analgesia have been identified in the midbrain of the rat (Cannon, Prieto, Lee,

This research was part of a doctoral dissertation conducted in the Department of Psychology, Case Western Reserve University. The author wishes to thank Robert C. Wilcott, who served as her advisor. Reprint requests should be directed to the author at: Department of Psychology, Case Western Reserve University, Cleveland, $\mathrm{OH} 44106$.
\& Liebeskind, 1982). Nonopioid analgesia appears to be induced by stressors, such as cold water swims (Walker, Berntson, Paulucci, \& Champney, 1981), and is often referred to as stress-induced analgesia (SIA). Short durations of stress appear to cause nonopioid analgesia, whereas longer durations induce opioid mechanisms (Akil et al., 1984). It has been suggested that both opioid and nonopioid systems may be involved in SPA (Akil et al., 1984).

Previous work on the rat has shown that strong analgesia that is opiate-based can be induced by stimulation at sites in the midbrain central gray and nearby regions (Mayer \& Liebeskind, 1974). Degree of analgesia varies, with the tail being more analgesic than the limbs (Soper, 1976). Only a few other behavioral effects accompanying SPA have been reported. During stimulation, sniffing (Balagura \& Ralph, 1973), an increase in respiration (Reynolds, 1969; Rhodes \& Liebeskind, 1978), and various kinds of aversive and escape behaviors (Reynolds, 1969; Rhodes \& Liebeskind, 1978) have been observed. Following stimulation, eating (Soper, 1976), orienting reflexes, and response to light touch or loud noises (Balagura \& Ralph, 1973) appear unimpaired. Earlier studies (Mayer \& Liebeskind, 1974; Reynolds, 1969) reported no overt seizures or changes in the cortical electroencephalogram (EEG), but later work (Rhodes \& Liebeskind, 1978) revealed the presence of both overt seizures and some high-voltage spiking occurring in the cortex or at the site of stimulation.

In the research presented here, it was found that, with the rat, SPA was always accompanied by a strong physiological and behavioral suppression. Suppressive effects included a reduction in locomotor activity, relaxation of skeletal muscle, reduction in autonomic activity, and a slowing of cortical EEG waves (indicative of drowsiness). These effects, along with the analgesia, outlasted the period of stimulation, and the suppression persisted over time even when the analgesia was no longer apparent.

Little attention has been paid to these suppressive effects by others studying SPA. However, it has been 
reported that similar responses often occur following painful footshock (Fanselow, 1980; Fanselow \& Bolles, 1979), and it has been suggested that they may be related to recuperative behaviors following a painful experience (Bolles \& Fanselow, 1980). Two experiments are described which were intended to assess these suppressive effects.

\section{EXPERIMENT 1}

The purpose of this experiment was to describe the physiological and behavioral suppression accompanying SPA in the periaqueductal gray (PAG). The physiological parameters investigated were heart rate and EEG. Behavioral parameters were locomotor activity, general overt behavior, righting reflexes, and the ability of the animal to cling to and climb a wire grid. Analgesia was assessed by the tail-immersion test of Sewell and Spencer (1976) and a modification of the jump-flinch test of Bonnet and Peterson (1975).

\section{Method}

Animals. Twenty-nine male Holtzman rats, weighing 280 to $320 \mathrm{~g}$ at the time of surgery, were used as subjects. They were housed in individual cages and had free access to food and water.

Surgery. Anesthesia was induced by the administration of sodium pentobarbital $(50 \mathrm{mg} / \mathrm{kg}$, ip). For brain stimulation, bipolar electrodes (Plastic Products Co., Roanoke, VA), which were made of steeless steel wires $0.2 \mathrm{~mm}$ in diameter and insulated except at the cut tip, were used. One electrode was implanted in the left side of the brain in an area that was found to produce suppressive effects in pilot observations (approximately at the coordinates A-P 0.0) using the atlas of Pellegrino, Pellegrino, \& Cushman (1979). The second electrode was placed on the right side of the brain at coordinates between A-P +1.0 and -1.0 . This was done to explore the extent of the anatomical range of placements on the right side, while being reasonably sure of obtaining suppression from the left placements. In each case, the best electrode placement was used for data collection. Electrodes were secured to the cranium with dental acrylic, which was reinforced with three stainless steel jeweler's screws inserted into the bone.

For EEG recording, a single bipolar electrode was untwisted, the insulation was removed from the lower $1.5-\mathrm{mm}$ part of each wire, and each end was folded over to form a small loop. This was inserted beneath the bone onto the dura, with one end over the frontal cortex and the other end over the parietal cortex, both on the left side. They were secured to the cranium in the same way as the stimulation electrodes. EEG was recorded only on one side, because previous observations had shown that cortical EEG activity on both sides of the brain were apparently the same, and that fewer leads reduced the amount of restraint on the rat.

Heart rate electrodes consisted of stainless steel wires inserted beneath the skin in the midthoracic section of the subject's back on each side and attached to a miniature receptacle.

Histology. At the conclusion of testing, the rats were given lethal doses of sodium pentobarbital, and intracardiac perfusion was performed using $0.9 \%$ saline followed by neutral buffered Formalin $10 \%$. Excised brains were stored for $48 \mathrm{~h}$ in the Formalin solution and blocked at the angle specified in the atlas. Serial frozen sections $(50 \mu)$ were cut and placed on glass microscope slides, and left unstained. A projection microscope was used to verify electrode placements.

Electrical brain stimulation. Stimulation was done with a Grass S4 stimulator feeding through a Grass SIU5 stimulus isolation unit and a Grass CCU1 constant current unit. Brain stimulation consisted of $0.5-\mathrm{msec}$ square-wave pulses delivered at a frequency of $50 / \mathrm{sec}$ and presented for $10 \mathrm{sec}$ at current levels described below.

Analgesia testing. During the tail-immersion test (Sewell \& Spencer, 1976), each rat was placed in a Plexiglas cylindrical chamber (Fisher Scientific Co.) with the tail protruding freely from the rear of the chamber. Testing consisted of immersion of the tail in a constant water bath maintained at $55^{\circ} \mathrm{C}$, at an immersion depth of $10 \mathrm{~mm}$. Nociceptive reaction time (in seconds) was determined when the tip of the tail was removed from the hot water by a characteristic sudden "flick" of the tail. A 15-sec cutoff time was set to prevent tissue damage to the tail. Testing began immediately following termination of the stimulation, and this procedure was repeated once every 2 min thereafter until the tail-flick latency had returned to baseline. A baseline tail-flick threshold was established $60 \mathrm{~min}$ prior to brain stimulation. Pre- and poststimulation responses were compared to determine degree of analgesia.

For the jump-flinch test (Bonnet \& Peterson, 1975), the rat was placed in a modified Skinner box (Gerbrand $\mathrm{Co}$.) with the bar and feeder removed. This was housed inside an observation chamber (Lehigh Valley Products Co.). A one-way window in the door of the observation chamber allowed for viewing while testing. The jump-flinch test was modified so that only five categories of pain threshold were used:(1) elevation of one or two paws from the grid floor, (2) elevation of three or four paws in a variety of configurations, (3) elevation of four paws simultaneously, (4) running, and (5) jump-elevation of four paws simultaneously in excess of 2 in. off the grid floor. This method of scoring was used because it had been found, both in pilot observations and in testing of surgically untreated laboratory controls, that, in this strain of rats, the flinch was not reliably obtained; also, the oscillation and tetanic movements observed by Bonnet and Peterson (1975) were not observed at all, but were replaced by running. Furthermore, a proportionally reasonable increase in current always elicited a sudden "jump" in excess of $2 \mathrm{in}$. from the grid floor. It was not determined whether these response differences were due to our laboratory equipment or were peculiar to this particular strain of rats. Shock was delivered by a constant-voltage variable-shock source (Lafayette Instruments) through the grid floor of the Skinner box, to which a shock scrambler (Lafayette) was coupled. Shock was delivered at $60 \mathrm{~Hz}$ ac for a $200-\mathrm{msec}$ duration with a 20 -sec intershock interval. Shocks were delivered at increasing intensities until the behavior of each response category was observed. For scoring, the shock intensity of the five categories was totaled, and the mean intensity value was used as the pain threshold. A baseline footshock threshold was established $60 \mathrm{~min}$ prior to brain stimulation. Preand poststimulation responses were compared to determine degree of analgesia.

Test for behavioral suppression. Behavioral observations were made by viewing the rat through the one-way window in the door of the observation chamber. Following stimulation, behavior was rated as follows: (1) frozen-except for breathing and eye blink, no movement at all, not even a whisker twitch (Fanselow, 1980); (2) resting-lying down, with movement of head or paw only for postural adjustment; (3) quiet-occasional change of position, no grooming; and (4) normal activity-including walking, sniffing, exploring, and grooming. If a rat displayed a behavior that belonged to a higher rating level (e.g., grooming), it was assigned the higher rating for that entire interval. To assure rater reliability of behavioral observations, two observers independently rated the behavioral activity of five rats following stimulation. No differences were found between the ratings of the two observers.

Handling observations were made during the second testing session for behavioral suppression (described below). A pinch to the tail was employed to determine if the animal was able to respond to a painful stimulus and to avoid mislabeling suppression as analgesia. Two other behaviors were noted: (1) righting reflexes, and (2) the ability of the rat to cling to and climb the side of a wire 
grid placed at a $45^{\circ}$ angle. The wire grid consisted of the bottom of a standard rat cage tilted to a $45^{\circ}$ angle (Jaquet \& Marks, 1976).

Tests for physiological suppression. Heart rate recordings were made on a Grass Model 5D polygraph. Recording equipment was turned off during stimulation and turned on again immediately following stimulation. Heart rate was counted as beats per minute.

EEG recordings were made on a second channel of the Grass polygraph. EEG changes were assessed using the method of Robinson (1980). Briefly, this was done by using a clear plastic ruler to measure the peak-to-peak amplitude (in millimeters) of the largest single rhythmic slow-activity (RSA) waves that lasted at least $1 \mathrm{sec}$. Measurements were taken from all EEG waves in each record, but only the largest RSA waves are reported along with the frequency and interval fluctuations.

Procedure. Two weeks following surgery, the rat was placed in the Skinner box, and each electrode site was pretested to determine the current intensity to be used at that site. Although analgesia is known to be a function of current intensity (Cannon et al., 1982), suppression appeared to be an all-or-none occurrence. Preliminary observations had indicated that suppression of the other variables was present if heart rate reduction occurred. Since heart rate was the easiest to determine, the criteria for current intensity was that which would produce at least a $25 \%$ reduction in heart rate within the first $2 \mathrm{~min}$ poststimulation. Current levels began at $0.05 \mathrm{~mA}$ and increased in steps of $0.03 \mathrm{~mA}$, with the maximum at $0.15 \mathrm{~mA}$. Pilot data had indicated that if suppression was not present at this latter current intensity, it would not be obtained at all. To reduce variability in analgesia, the final current intensity range was set between 0.10 and $0.15 \mathrm{~mA}$ for all analgesia and suppression testing sessions. During the pretesting session, many rats displayed aversive reactions to the stimulation, as had been described by others (Balagura \& Ralph, 1973; Mayer \& Liebeskind, 1974; Reynolds, 1969; Soper, 1976). These rats were set aside and tested separately as described below.

Each brain site was tested at five test periods spaced 1 week apart: (1) pretesting for the current intensity, (2) tail-immersion test, (3) jump-flinch test, (4) test for suppression and (5) a second test for suppression, including handling observations.

For Test 4 (suppression), the procedure was as follows: On the day of testing, a baseline heart rate and EEG record was made for $8 \mathrm{~min}$ for each rat prior to testing. Before testing, all rats displayed "'normal activity" and were given a baseline rating of 4 . Brain stimulation was then presented for $10 \mathrm{sec}$. Immediately following stimulation, recording equipment was turned on, and recordings resumed continuously for a $10-\mathrm{min}$ period. Heart rate, EEG, and behavior were scored for each 1 -min interval for the first $10 \mathrm{~min}$ poststimulation. After the first $10 \mathrm{~min}$, scoring was as follows: Behavior was observed continuously during the entire 60 -min testing period, and a rating score was given for each 5-min interval. A sample heart rate and EEG record was made for $1 \mathrm{~min}$ at the end of each successive 5-min interval until the 60-min time limit had been reached. For each variable tested, the mean values of the 12 poststimulation 5 -min intervals were totaled, and a mean value was obtained as an overall score. This mean value was compared with the prestimulation value to determine percent change.

Testing at sites producing aversive behaviors. Brain sites that evoked aversive behaviors during stimulation were tested separately. During stimulation, at current levels about $0.08 \mathrm{~mA}$, the rats displayed behaviors such as squeaking, running, circling, and jumping. Reducing the current intensity to $0.05 \mathrm{~mA}$ produced suppression without these aversive reactions. Therefore, these sites were tested for suppression at the $0.05 \mathrm{~mA}$. Because pilot observations had revealed that suppression was often obtained without analgessia at nonaversion sites, it was expected that some but not other sites might show analgesia. Therefore, in order to assess only the presence or absence of analgesia at these aversion sites, the tailimmersion test was used at a current of $0.10 \mathrm{~mA}$, which was approximately the same current used at nonaversion sites. This test could be done because the rat was restrained in the Plexiglas cylinder.

\section{Results}

Anatomy. The locations of 29 sites tested are shown in Figure 1. During stimulation, behavior was greatly reduced at 14 sites (sites 1 through 14). After stimulation, analgesia and suppression (AS) were present at 8 of these sites (sites 1 through 8); suppression only (SO) was present at 6 of these sites (sites 9 through 14). In general, the SO sites were located in the dorsal PAG, whereas the AS sites were either close to the aqueduct or in the ventromedial region. During stimulation at 12 sites, aversive reactions (AR) occurred, and for each of these sites, analgesia and suppression was present following stimulation (sites 15 through 26). Stimulation at the remaining 3 sites produced no observable effects (sites 27 through 29). Site 27 was located in a region far from the PAG, and sites 28 and 29 were on the dorsolateral peripheral border of the PAG and touching other structures.

Analgesia. Analgesia data are presented in Table 1. As can be seen, stimulation at AS sites (1-8) produced significant increases in pain thresholds with both tests, whereas stimulation at SO sites (9-14) produced little effects on pain thresholds with either test.

Behavioral suppression. Significant behavioral suppression was observed at all AS and SO sites. This suppression occurred within the first $5 \mathrm{sec}$ following stimulation and remained fairly consistent over the entire 60 min testing period. Rats appeared quiet, and some were motionless. The whiskers did not move, and spontaneous locomotor activity was abolished. They appeared "frozen." Some rats showed only an occasional change of position. Behavior ratings are given in Figure 2a. As can be seen, the greatest reduction in activity occurred during the first 5 min poststimulation. For the AS group $(\mathrm{N}=8)$, a mean reduction in behavior ratings of $31.7 \%$ occurred. Means before and after stimulation were 4 and 2.73 (two-tailed $t$ test) $[t(7)=7.92, p<.001]$. For the SO group $(\mathrm{N}=6)$, a mean reduction in activity of $35.2 \%$ occurred. Means before and after were 4 and 2.59 [t $(5)$ $=9.56, \mathrm{p}<.001]$. When the mean difference before and after stimulation of the AS group was compared with that of the SO group, the difference between the two groups was not significant $[t(12)=0.721, p>.05]$.

Handling observations revealed signs of muscle relaxation. The rats showed no resistance to handling, and some appeared as if anesthetized. Righting reflexes were present in all rats, and all rats could cling to and climb the wire grid placed at a $45^{\circ}$ angle, indicating that the reduction in activity was not part of a generalized motor debilitation. The SO group responded to tailpinch with a normal withdrawal response, whereas the AS group did not respond, as was expected, since they were the analgesic group.

Physiological suppression. Heart rate was dramatically reduced following stimulation, as shown in Figure $2 b$. The AS group $(\mathrm{N}=6)$ showed an overall reduction of $12.7 \%$ following stimulation. The mean heart rates (beats/minute) before and after stimulation for the AS group were 416 and $364[\mathrm{t}(5)=8.267, \mathrm{p}<.01]$. The 


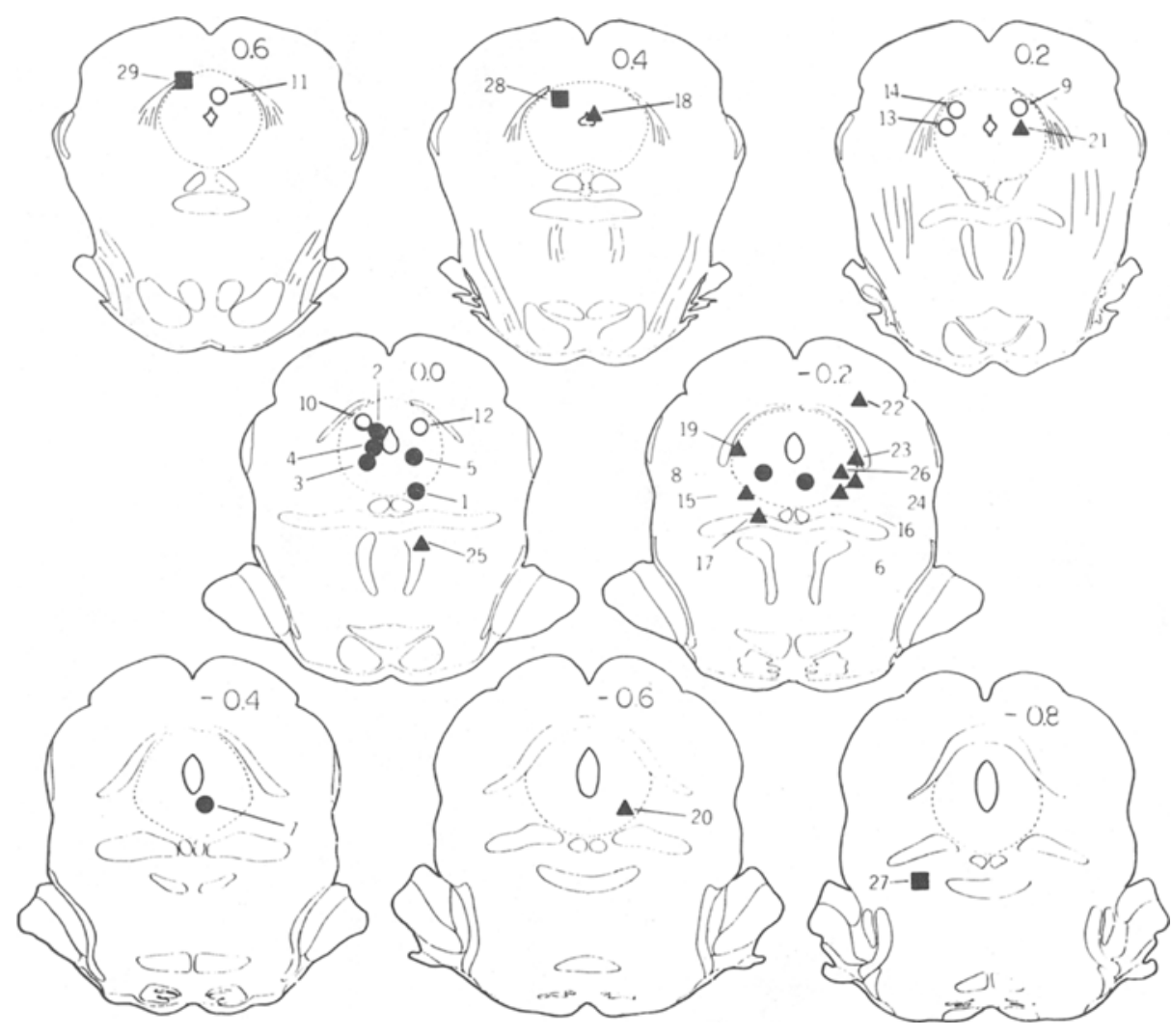

Figure 1. Reconstruction of midbrain sites that produced analgesia and suppression (closed circles), suppression only (open circles), aversive reactions, analgesia and suppression (triangles), and of sites that produced no effect (squares). Drawings modified from the atlas of Pellegrino, Pellegrino, and Cushman (1979).

Table 1

Individual Pain Thresholds Before and After Central Gray Stimulation and Group Means for AS Sites (1-8) and SO Sites (9-14)

\begin{tabular}{|c|c|c|c|c|c|c|c|c|c|c|}
\hline \multirow[b]{2}{*}{ Site } & \multicolumn{6}{|c|}{$\begin{array}{c}\text { Mean Tail-Flick Latencies (sec) } \\
\text { (Tail-Immersion Test) }\end{array}$} & \multicolumn{4}{|c|}{$\begin{array}{c}\text { Mean Footshock Thresholds (mA) } \\
\text { (Jump-Flinch Test) }\end{array}$} \\
\hline & $\begin{array}{l}\text { Current } \\
(\mathrm{mA})\end{array}$ & Before & After & Difference & $\begin{array}{l}\text { Percent } \\
\text { Change }\end{array}$ & $\begin{array}{c}\text { Duration } \\
\text { of Change }\end{array}$ & Before & After & Difference & $\begin{array}{l}\text { Percent } \\
\text { Change } \\
\end{array}$ \\
\hline 1 & 0.15 & 4.5 & 8.3 & 3.8 & 84.4 & $20.0 \mathrm{~min}$ & 2.29 & 3.37 & 1.08 & 47.2 \\
\hline 2 & 0.10 & 6.5 & 15.0 & 8.5 & 130.7 & $30.0 \mathrm{~min}$ & 2.12 & 4.26 & 2.14 & 100.9 \\
\hline 3 & 0.15 & 4.7 & 14.7 & 10.0 & 212.8 & $75.0 \mathrm{~min}$ & 1.69 & 2.49 & 0.80 & 47.3 \\
\hline 4 & 0.10 & 4.0 & 15.0 & 11.0 & 275.0 & $30.0 \mathrm{~min}$ & 2.74 & 7.52 & 4.78 & 174.5 \\
\hline 5 & 0.10 & 4.0 & 15.0 & 11.0 & 275.0 & $25.0 \mathrm{~min}$ & 1.60 & 3.47 & 1.87 & 116.9 \\
\hline 6 & 0.15 & 3.7 & 7.2 & 3.5 & 94.6 & $12.0 \mathrm{~min}$ & 2.07 & 2.57 & 0.50 & 24.2 \\
\hline 7 & 0.10 & 7.0 & 9.5 & 2.5 & 35.7 & $12.0 \mathrm{~min}$ & 2.97 & 3.47 & 0.50 & 16.8 \\
\hline \multirow[t]{2}{*}{8} & 0.10 & 3.2 & 8.3 & 5.1 & 159.4 & $5.0 \mathrm{~min}$ & 2.66 & 3.44 & 0.78 & 29.3 \\
\hline & & 4.7 & 11.6 & $6.9^{*}$ & 147.3 & $36.5 \mathrm{~min}$ & 2.26 & 3.82 & $1.56^{* *}$ & 64.8 \\
\hline 9 & 0.10 & 6.5 & 6.5 & 0.0 & 0.0 & none & 1.95 & 1.85 & -0.10 & -5.1 \\
\hline 10 & 0.15 & 4.0 & 2.9 & -1.1 & -26.5 & none & 1.64 & 1.30 & -0.28 & -17.0 \\
\hline 11 & 0.15 & 4.4 & 5.2 & 0.8 & 18.0 & none & 2.27 & 2.50 & 0.23 & 10.0 \\
\hline 12 & 0.15 & 5.9 & 5.2 & -0.7 & -12.5 & none & 2.08 & 2.10 & 0.02 & 0.9 \\
\hline 13 & 0.10 & 4.8 & 4.0 & -0.8 & -16.7 & none & 2.69 & 2.68 & -0.01 & -0.4 \\
\hline \multirow[t]{2}{*}{14} & 0.10 & 4.7 & 4.0 & -0.7 & -14.9 & none & 1.87 & 1.70 & -0.17 & -9.0 \\
\hline & & 5.1 & 4.6 & $0.4 \dagger$ & -8.0 & & 2.08 & 2.03 & $-0.05 \uparrow \uparrow$ & -2.5 \\
\hline
\end{tabular}



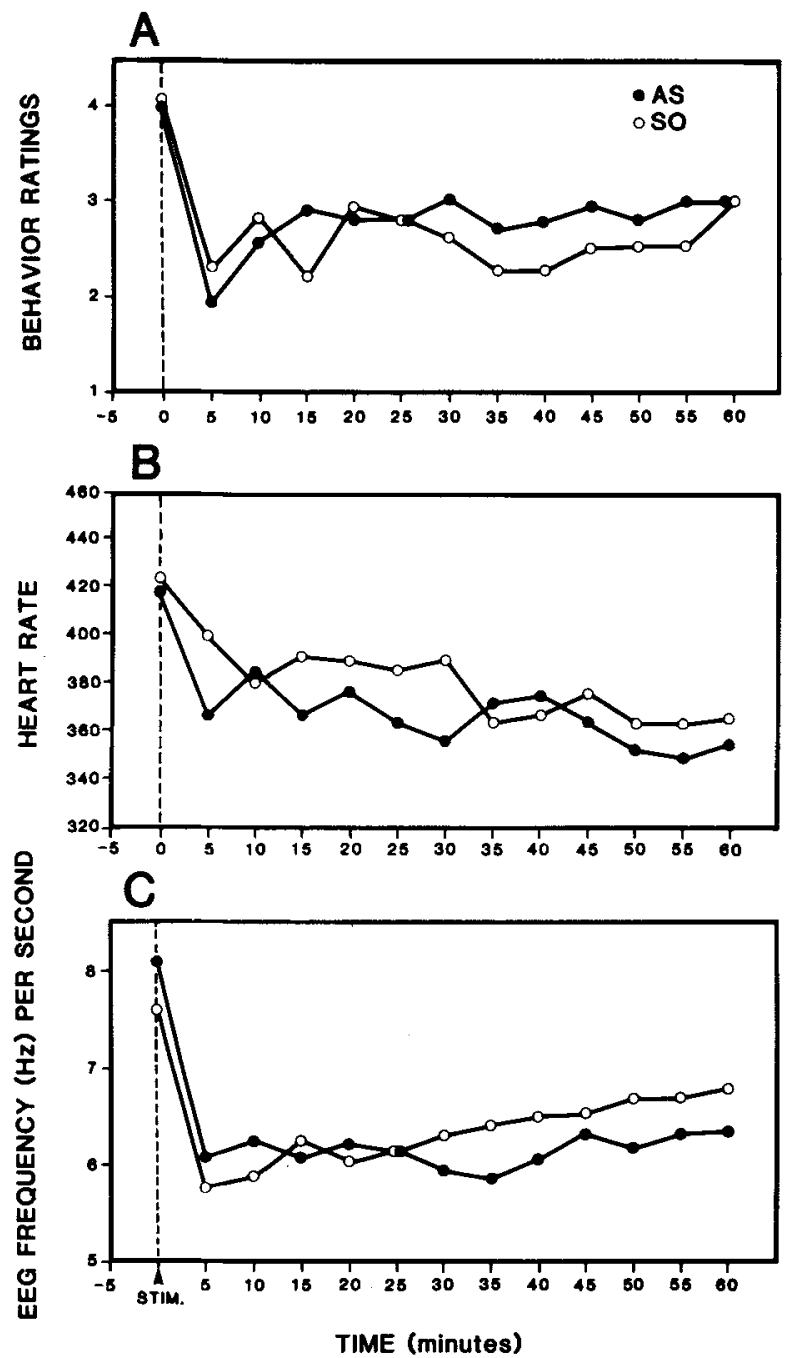

Figure 2. Physiological and behavioral suppression following electrical stimulation of the central gray. Each point on the graph represents the mean value for each preceding 5-min interval. Zero point represents the mean value of the 5-min baseline period immediately before stimulation. (A) Overt behavior ratings. $1=$ frozen, $2=$ resting, 3 = quiet, $4=$ normal activity. (B) Changes in heart rate. (C) Changes in cortical EEG frequency.

SO group $(\mathrm{N}=5)$ showed an overall reduction in heart rate of $10.7 \%$. The means before and after were 424 and $378[\mathrm{t}(4)=5.80, \mathrm{p}<.01]$. When the mean difference before and after stimulation of the AS group was compared with that of the SO group, the difference between the two groups was not significant $[\mathrm{t}(9)=0.511, \mathrm{p}>$ $.05]$. (The smaller $\mathrm{N}$ reported for heart rate is due to equipment failure.)

EEG frequency was significantly reduced following stimulation at all AS and SO sites, as shown in Figure 2c. For the AS group ( $\mathrm{N}=8)$, a mean reduction of $25 \%$ occurred. The means (in hertz) before and after stimulation were 8.15 and $6.11[\mathrm{t}(7)=3.85, \mathrm{p}<.01]$. For the SO group $(\mathrm{N}=6)$, a mean reduction of $19.8 \%$ occurred. The means before and after were 7.58 and $6.08[\mathrm{t}(5)=3.318$, $\mathrm{p}<.05]$. When the AS group and the SO group were compared, no significant difference was found between the two groups $[\mathrm{t}(12)=1.735, \mathrm{p}>.05]$.

Aversion response sites. The presence of aversive reactions (AR) during stimulation did not prevent the appearance of either the analgesia or suppressive effects after stimulation. For the AR group ( $\mathrm{N}=12)$, analgesia obtained in the tail-immersion test was comparable to that obtained with the AS group. The AR group showed an $88 \%$ mean increase in tail-flick latencies poststimulation. The means before and after stimulation were 4.4 and $8.44 \sec [t(11)=3.188, p<.01]$. However, the duration of the analgesia was from 2 to $15 \mathrm{~min}$, which was shorter than that for the AS group.

Aversion sites also showed similar physiological and behavioral suppression after stimulation, despite the fact that they were tested at a low current $(0.05 \mathrm{~mA})$. They showed a $27.8 \%$ mean reduction in overt behavior following stimulation. The means before and after stimulation were 4 and 2.88 [t(11) $=4.986, \mathrm{p}<.001]$.

The AR group showed a mean heart rate reduction of $5.1 \%$ following stimulation. The mean heart rates before and after stimulation were 410 and $389(\mathrm{~N}=12)$ [t(11) $=2.49, \mathrm{p}<.05]$. Two aversion sites produced atypical poststimulation heart rate records, with an increase in heart rate during the first 5-min period.

EEG changes for the AR group resembled those of the other groups. For the AR sites, there was a mean reduction in frequency of $12 \%$. The means before and after 7.6 and $6.7 \mathrm{~Hz}(\mathrm{~N}=12)[\mathrm{t}(11)=3.166, \mathrm{p}<.01]$.

\section{EXPERIMENT 2}

The observation that suppressive effects can be evoked independently of analgesia at some electrode sites, suggests that the suppression may not be an opiate effect, but be mediated by some other mechanism. This experiment was done to determine if the suppression was an opiate effect. If it was, then it should be sensitive to naloxone blockade (Akil et al., 1976; Pert \& Snyder, 1973a; Snyder, Enna, \& Young, 1977). If it was not sensitive to naloxone, then it would be controlled by a separate, but perhaps related, mechanism.

\section{Method}

Animals. Results were obtained from 15 male Holtzman rats, which were used in Experiment 1. Five of these rats had AS sites, 4, SO sites, and 6, AR sites.

Procedure. EEG, heart rate, and behavior ratings were measured and scored the same way as in Experiment 1. The rats were placed inside the observation chamber, and a baseline EEG, heart rate, and behavior rating was established for each rat for $8 \mathrm{~min}$ prior to testing. The rat was then administered naloxone $(1 \mathrm{mg} / \mathrm{kg}$ ip), and was placed back in the observation chamber. Recordings and behavior ratings continued for a $15-\mathrm{min}$ period in order to determine whether any changes resulted from the injection procedure. No changes were found. Brain stimulation was administered $15 \mathrm{~min}$ 
postinjection. Recordings and behavior ratings proceeded as in Experiment 1. However, in Experiment 2, the preinjection baseline was compared with poststimulation responses to determine percent change. Naloxone blockage of analgesia was not tested because it had been well documented (Mayer \& Hayes, 1975; Akil et al., 1976).

\section{Results}

AS and SO sites. Naloxone did not block the suppressive effects produced by stimulation in any of the sites tested. The reduction in behavior ratings was unaffected by naloxone. With the AS group $(\mathrm{N}=5)$, a mean reduction of $32 \%$ occurred. The means before and after were 4 and $2.72[\mathrm{t}(4)=6.936, \mathrm{p}<.01]$. The SO group $(\mathrm{N}=4)$ showed similar results with a mean reduction of $35 \%$. The means before and after were 4 and $2.56[\mathrm{t}(3)$ $=6.76, \mathrm{p}<.01]$.

Heart rate was also unaffected by naloxone. With the AS group $(N=4)$, a mean heart rate reduction of $13.9 \%$ occurred. The means before and after were 429 and 364 $[\mathrm{t}(3)=5.172, \mathrm{p}<.02]$. For the SO group $(\mathrm{N}=4)$, a mean heart rate reduction of $13.2 \%$ occurred. The means before and after were 400 and $347[\mathrm{t}(3)=3.186, \mathrm{p}<$ .05].

Cortical EEG changes produced by stimulation were not affected by naloxone at any of the sites tested. A typical EEG record is given in Figure 3, which shows a sample of a baseline EEG, EEG after stimulation, and EEG after naloxone pretreatment. EEG frequency for the AS group $(\mathrm{N}=5)$ showed a mean reduction of $30.4 \%$. The means before and after were 7.5 and $5.2[t(4)=5.61$, $\mathrm{p}<.01]$. For the $\mathrm{SO}$ group $(\mathrm{N}=4)$, a mean reduction of $19.5 \%$ occurred. The means before and after were 7 and $5.63[\mathrm{t}(3)=6.013, \mathrm{p}<.01]$.

Aversion response sites. Naloxone also did not block any of the suppressive effects from sites which evoked aversion responses. For the AR group $(N=6)$, the mean reduction in behavior ratings was $30.5 \%$ in the naloxone condition. The means before and after were 4 and 2.98 $[\mathrm{t}(5)=4.811, \mathrm{p}<.01]$.

Heart rate was reduced $8.7 \%$ in the naloxone condition for the AR group. The means before and after were 401 and $366[\mathrm{t}(5)=13.53, \mathrm{p}<.001]$.

Cortical EEG frequency for the AR group showed a mean reduction in the naloxone condition of $10.1 \%$. The means before and after were 6.4 and $5.91 \mathrm{~Hz}[\mathrm{t}(5)=$ $2.955, \mathrm{p}<.05]$.

\section{DISCUSSION}

Experiment 1 demonstrated that stimulation of the PAG and nearby regions can produce both analgesia and a strong physiological and behavioral suppression. The suppression can be obtained independently of analgesia by stimulation at dorsal PAG sites. Traditionally, and in this paper, dorsal is defined as the region of the mesencephalon that lies dorsal to the sulcus limitans, the anatomical longitudinal groove which delimits the embryonic alar and
1

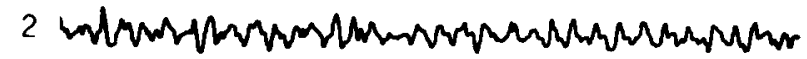

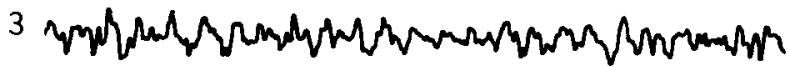

$\underset{1 \operatorname{SEC}}{1} 50 \mu \mathrm{V}$

Figure 3. Typical cortical EEG record (1) before stimulation, (2) after stimulation, and (3) after stimulation with naloxone pretreatment.

basal plates (Brodal, 1981). A different line of demarcation dividing dorsal and ventral zones has been defined by others (Cannon et al., 1982; Prieto, Cannon, \& Liebeskind, 1983), and these distinctions should be considered when comparing results. Further, sites that evoked analgesia and suppression were located near or touching the border of the aqueduct itself and not necessarily in or near the nucleus raphe magnus as described by others (e.g., Akil et al., 1976; Mayer, 1980; Mayer \& Liebeskind, 1974).

Stimulation can also produce aversive reactions at some PAG sites. As shown in Figure 1, electrode sites that produced aversive reactions, analgesia, and suppression were located on the lateral peripheral border of the PAG. None of these aversion sites produced suppression independently of analgesia, despite the fact that many aversion sites had dorsal loci. Other workers have reported similar aversive responses and analgesia obtained with stimulation at peripheral sites (e.g., Balagura \& Ralph, 1973; Mayer \& Liebeskind, 1974; Rhodes \& Liebeskind, 1978). These workers proposed that there was an overlapping zone located on the lateral PAG border which would produce both analgesia and aversion. This zone of aversion has been demonstrated to be proportionally greater in the cat (Liebeskind et al., 1973). In the present work, although analgesia was obtained at lateral aversion sites, it was of considerably shorter duration than that obtained at more medial placements.

Experiment 2 demonstrated that the suppressive effects were insensitive to naloxone blockade, and therefore not an opiate-based phenomenon. Furthermore, naloxone $(1 \mathrm{mg} / \mathrm{kg}$ ) did not change the magnitude or duration of these effects, nor did it reduce spontaneous activity at either AS or SO sites. This is in contrast to the observations of Walker and his co-workers (1981), who reported that a $2-\mathrm{mg} / \mathrm{kg}$ dose of naloxone reduces spontaneous activity in rats under both baseline conditions and conditions of stress (e.g., stress-induced analgesia).

It has been suggested that a second analgesia system, which is naloxone-insensitive, is present in the midbrain of the rat (Cannon et al., 1982). Recently, electrophysiological studies have demonstrated that morphine and meperidine (Demerol) excite different pathways in the brain (Emmers, 1981). Further, it has been shown that SPA is more similar to meperidine analgesia than it is to 
morphine analgesia (Emmers, 1981), but during SPA both an opiate-based analgesia system and a meperidine system may be activated simultaneously. Such an overlapping system may explain why some workers have found that naloxone reversal of SPA is only partial (Mayer \& Liebeskind, 1974: Rhodes \& Liebeskind, 1978), since meperidine is naloxone insensitive (Goodman, Gilman, \& Gilman, 1980).

The finding that suppression can be evoked independently of analgesia suggests that an endogenous tranquilizing system that is neuroanatomically separated from SPA may exist. Mountcastle (1980) has suggested that a reaction to pain consists of at least three main components: (1) the perception of the sensation of pain, (2) the autonomic reflexive actions which alert the animal and prepare him to "fight or flight," and (3) the reflexive actions of somatic musculature that enable him to perform the appropriate behavioral response that will lead to the termination of the painful event (attack or run away). If a painful event is accompanied by anxiety and muscie tension, then it is reasonable to assume that an endogenous pain control system should comprise substances and mechanisms that would alleviate pain, decrease muscle tension, and calm the organism. Such a mechanism of sedation could serve the animal under conditions in which pain is not a factor, such as in a fearful or stressful situation. Speculation about the chemical basis for such a tranquilizing system is beyond the scope of this paper, but recently it has been reported that there is a benzodiazepinereceptor ligand that exists in nervous tissue (Reichter, 1981; Snyder et al., 1977). Behavioral evidence for the existence of an endogenous tranquilizing system comes from workers studying brain and behavior mechanisms in fear and pain. Bolles and Fanselow (1980) have proposed a perceptual-defensive-recuperative (PDR) model of fear and pain, which assumes that fear and pain are motivational systems and that, under certain circumstances, fear can inhibit pain (via the endorphins) while defensive behaviors are being organized and coordinated. Upon termination of the painful event, recuperative behaviors ensue. The physiological and behavioral suppression observed with SPA in the present work parallels the "recuperative phase" of the PDR model and appears to support it.

\section{REFERENCES}

ADAms, J. E. (1976). Naloxone reversal of analgesia produced by electrical stimulation in the human. Pain, 2, 161-166.

AKIL, H., MAYer, D. J., \& LiEbeskind, J. C. (1976). Antagonism of stimulation-produced analgesia by naloxone, a narcotic antagonist. Science, 191, 961-962.

Akil, H., Watson, E. Y., Lewis, M. E., Khachaturian, H., \& WALKER, M. J. (1984). Endogenous opioids: Biology and function. Annual Review of Neuroscience, 7, 223-255.

Anderson, S. D., Basbaum, A. I. \& Fields, H. L. (1977). Response of medullary raphe neurons to peripheral stimulation and to systemic opiates. Brain Research, 123, 363-368.
Balagura, S., \& RalPh, T. (1973). The analgesic effect of electrical stimulation of the diencephalon and mesencephalon. Brain Research, 60, 369-379.

Basbaum, A. I., Clanton, C. H., \& Fields, H. L. (1976). Opiate and stimulus-produced analgesia: Functional anatomy of a medullo spinal pathway. Proceedings of the National Academy of Science, 73, 4685-4688.

Bolles, R. C., \& Fanselow, M. S. (1980). A perceptual-defensiverecuperative model of fear and pain. Behavioral and Brain Sciences, 3, 291-323.

Bonnet, K. A., \& Peterson, K. E. (1975). A modification of the jumpflinch technique for measuring pain sensitivity in rats. Pharmacology, Biochemistry and Behavior, 3, 47-55.

Bowsher, D. (1957). Termination of the central pain pathway in man: The conscious appreciation of pain. Brain, 80, 606-625.

Brodal, A. (1981). Neurological anatomy: In relation to clinical medicine (3rd ed.). New York: Oxford University Press.

Cannon, J. T., Prieto, G. J., Lee, A., \& Liebeskind, J. C. (1982). Evidence for opioid and non-opioid forms of stimulation-produced analgesia in the rat. Brain Research, 43, 315-321.

Emmers, R. (1981). Pain: A spike-interval coded message in the brain. New York: Raven Press.

Fanselow, M. S. (1980). Conditional and unconditional components of post-shock freezing. Pavlovian Journal of Biological Science, 15, 177-182.

FANSElow, M. S., \& Bolles, R. C. (1979). Naloxone and shock-elicited freezing in the rat. Journal of Comparative and Physiological Psychology, 93, 736-744.

GeIsLeR, G. J., \& Liebeskind, J. C. (1976). Inhibition of visceral pain by electrical stimulation of the periaqueductal gray matter. Pain, 2, 43-48.

Goodman, A. G., Gilman, L. S., \& Gilman, A. (1980). Goodman and Gilman's: The pharmacological basis for therapeutics. New York: MacMillan.

Hosobuchi, Y., Adams, J. E., \& Linchitz, R. (1977). Pain relief by electrical stimulation of the central gray matter in humans and its reversal by naloxone. Science, 197, 183-186.

Hughes, J. T., Smith, T. W., \& Kosterlitz, H. W. (1975). Identification of two related pentapeptides from the brain with potent opiate agonist activity. Nature, 258, 577-579.

JAQUET, Y. F., \& MARKS, N. (1976). The C-fragment of $\beta$-lipotrophen: An endogenous neuroleptic or antipsychogen? Science, 194, 632-634.

Katz, R. J., CARroll, B. J., \& Baldright, G. (1978). Behavioral activation by enkephalins in mice. Pharmacology, Biochemistry and Behavior, 8, 493-496.

Liebeskind, J. C., Guilband, G., Besson, M. M., \& Oliveras, J. L. (1973). Analgesia from electrical stimulation of the periaqueductal gray matter in the cat: Behavioral observations and inhibitory effects on spinal cord interneurons. Brain Research, 54, 441-446.

MAYER, D. J. (1980). Endogenous analgesia systems: Neural and behavioral mechanisms. In J. J. Bonica (Ed.), Advances in pain research and therapy (Vol. 3, pp. 345-410). New York: Raven Press.

MAYER, D. J., \& HAYES, R. (1975). Stimulation-produced analgesia: Development of tolerance and cross-tolerance to morphine. Science, 188, 941-943.

MAYeR, D. J., \& Liebeskind, J. C. (1974). Pain reduction by focal electrical stimulation of the brain: An anatomical and behavioral analysis. Brain Research, 68, 73-95.

MountCastle, V. B. (1980). Medical physiology (14th ed., Vol. 1, pp. 391-427). St Louis: Mosby.

Pellegrino, L. J., Pellegrino, A. S., \& Cushman, A. J. (1979). A stereotaxic atlas of the rat brain. New York: Plenum Press.

PerT, C. B., \& SNYder, S. H. (1973a). Opiate receptor: Demonstration in nervous tissue. Science, 197, 1011-1014.

PerT, C. B., \& SNYDER, S. H. (1973b). Properties of opiate receptor binding in rat brain. Proceedings of the National Academy of Science, 70, 2245-2247.

Prieto, G. J., Cannon, J. T., \& Liebeskind, J. C. (1983). N. raphe magnus lesions disrupt stimulation-produced analgesia from ventral 
but not dorsal midbrain areas in the rat. Brain Research, 261, 53-57. Reichter, J. J. (1981). Current theories about the mechanisms of action of bensodiazepines and neuroleptic drugs. Anesthesiology, 54, 66-72.

REYNOLDS, D. V. (1969). Surgery in the rat during electrical analgesia induced by focal brain stimulation. Science, 164, 444-445.

Rhodes, D. L., \& Liebeskind, J. C. (1978). Analgesia from rostral brain stimulation in the rat. Brain Research, 143, 521-532.

Richardson, D. E., \& AKIL, H. (1977a). Pain reduction by electrical brain stimulation in man. Part 1: Acute administration in periaqueductal and periventricular sites. Journal of Neurosurgery, 47, 178-183.

Richardson, D. E., \& AKIL, H. (1977b). Pain reduction by electrical brain stimulation in man. Part 2: Chronic self-administration in the periventricular gray matter. Journal of Neurosurgery, 47, 184-194.

RobInson, T. E. (1980). Hippocampal rhythmic slow activity (RSA: theta): A critical analysis of selected studies and discussion of possible species-differences. Brain Research Reviews, 2, 69-101.
Sewell, R. D. E., \& Spencer, P. S. J. (1976). Antinociceptive activity of narcotic agonists and partial agonist analgesics and other agents in the tail-immersion test in mice and rats. Neuropharmacology, 15, 683-688.

SNyder, S. H., EnNA, S. J., \& Young, A. B. (1977). Brain mechanisms associated with therapeutic actions of benzodiazepines: Focus on neurotransmitters. American Journal of Psychiatry, 134, 662-664. SOPER, W. Y. (1976). Effects of analgesic midbrain stimulation on reflexive withdrawal and thermal escape in the rat. Journal of Comparative and Physiological Psychology, 90, 91-101.

Walker, J. M., Berntson, G. G., Paulucci, T. S., \& Champney, T. C. (1981). Blockade of endogenous opiates reduces activity in the rat. Pharmacology, Biochemistry and Behavior, 14, 113-116.

(Manuscript received June 10, 1984; revision accepted for publication November 20, 1984.) 